Service social

\title{
Oppression amérindienne dans le contexte canadien
}

\section{Raymond Sioui}

Volume 40, numéro 3, 1991

Oppression, intolérance et intervention

URI : https://id.erudit.org/iderudit/706543ar

DOI : https://doi.org/10.7202/706543ar

Aller au sommaire du numéro

Éditeur(s)

École de service social de l'Université Laval

ISSN

1708-1734 (numérique)

Découvrir la revue

Citer cet article

Sioui, R. (1991). Oppression amérindienne dans le contexte canadien. Service social, 40(3), 42-52. https://doi.org/10.7202/706543ar

\section{Résumé de l'article}

L'histoire des contacts entre Amérindiens et non autochtones conduit aune oppression marquée du premier groupe. Cette oppression de toute évidence prend racine dans l'exercice d'un pouvoir davantage intéressé par

l'accaparement et la domination que par le respect des différences. Ce désir d'accaparement et de domination obnubilera le fait que l'Amérindien dispose d'une culture qui répond fort adéquatement à ses besoins et à son équilibre. Une justification officielle établissant a priori la supériorité d'une culture sur l'autre servira à soutenir un exercice visant à la domination, et même à donner à celle-ci un caractère de nécessité. Dans ce contexte, les contacts débuteront par des alliances de nécessité commerciale et militaire pour ensuite donner cours au légendaire processus des promesses rompues visant à faciliter l'occupation du territoire. Suivront des traités ou des lois dont l'esprit ne sera pas davantage respecté et une longue période d'infériorisation et de tentative d'assimilation. Peut-on s'étonner qu'un tel contexte donne lieu à une liste de symptômes typiques d'une oppression ? Peut-on aussi s'étonner d'une prise de conscience actuelle qui veut réhabiliter la culture amérindienne et permettre un meilleur contrôle sur son devenir?
Ce document est protégé par la loi sur le droit d'auteur. L'utilisation des services d’Érudit (y compris la reproduction) est assujettie à sa politique d'utilisation que vous pouvez consulter en ligne.

https://apropos.erudit.org/fr/usagers/politique-dutilisation/ 
Raymond Sioui, psychologue au Service de consultants autochtones enr.

\section{Oppression amérindienne dans le contexte canadien}

Raymond Sioui

Peut-on discuter d'oppression sans faire référence à l'exercice du pouvoir dans notre monde et à ses principales caractéristiques ? Pouvoir qui établit ses bases sur une trinité constituée de la finance (économique), du politique et du militaire. Une faiblesse en rapport à cette trinité et vous risquez davantage comme individu ou groupe de subir le pouvoir que de l'exercer.

Compétition, lutte pour la possession et l'accaparement sont les motivations d'un type de pouvoir qui semble avoir eu prédominance sur le désir du respect de l'autre et de l'équité. Si ce n'est la lutte entre individus ou classes sociales, alors ce sera la lutte entre nations, selon le palier où se joue ce pouvoir.

Ce que nous essaierons de comprendre, $c^{\prime}$ est comment ce type de pouvoir a pris racine et a évolué en terre d'Amérique. Dans cette histoire encore récente du contact entre Amérindiens et non-autochtones, nous tenterons de dégager les principales croyances véhiculées, ainsi que l'évolution des rapports de force entre ces deux groupes. Peut-être en arriverons-nous à extraire tout l'arbitraire et le relatif de ces lois, institutions, rapports de force et postulats économiques qui nous asservissent et qu'on nous propose le plus souvent à titre de vérités absolues, reliées à la bonne cause de l'évolution de la condition humaine.

\section{Avant l'influence des non-autochtones}

Le contexte amérindien, avant l'influence marquée du contact avec les non-autochtones, est un contexte d'autosuffisance et d'usu- 
fruit du territoire occupé. L'Amérindien évolue dans un cadre de grand respect pour la nature, à laquelle il attribue un caractère sacré. Le Dieu amérindien est reconnu dans et par son œuvre, sa création. L'Amérindien ne peut manquer respect à l'environnement sans déduire que c'est au Grand Esprit même qu'il manque respect. L'Amérindien de ce temps est davantage concerné par la spiritualité, par l'âme humaine que par la création extérieure (Élan Noir, 1987 : 9). L'homme tout entier, matière plastique de "l'artiste primordial ".

Pour les colonisateurs non autochtones, il en va tout autrement. Leur visée est très matérialiste et concerne l'agrandissement de leur royaume par l'accaparement de territoires et de leurs ressources premières. Pour ce faire, ils ont déjà élaboré certaines règles et lois qui définissent la procédure d'appropriation de territoires occupés. Ils ont plus particulièrement établi quelques critères sur lesquels baser la croyance en leur supériorité et y voir une justification de leurs actions de conquérants.

Si pour l'Amérindien l'accent est mis sur l'âme humaine, pour les rois du temps l'accent porte sur l'agrandissement de leur royaume, de leurs richesses matérielles et du pouvoir qu'ils en retirent. Deux entités se rencontrent pour lesquelles la figure et le fond de la gestalt semblent s'inverser.

Cela n'empêchera pas le colonisateur d'avoir ses évangélisateurs. II est de mise pour le roi de transformer les colonisés en bons sujets du roi et la religion peut servir de tremplin à cette fin. En ce sens, les religieux de ce temps sont déjà une sorte de fonctionnaires de l'État (ou royauté) et non uniquement de libres serviteurs de Dieu. Doit-on s'étonner qu'en pareille époque, pour bien intentionnés qu'ils aient pu être, ces religieux n'aient pour la plupart pu appréhender la réalité spirituelle du monde amérindien et y reconnaître un apport véritable à l'évolution de l'âme humaine?

La vérité en ces temps se veut unique en son expression, et l'époque ne se prête guère à l'ouverture sur d'autres cultures dont les modes de pensée et d'appréhension de la réalité sont différents. Cet autre différent ne peut et ne doit être qu'inférieur et dans l'erreur. Son sort est d'être ramené à la vérité : celle du colonisateur. Il en va de son salut, sous peine de disparaître pour ne pas entraver l'évolution de la condition humaine. L'intellect humain a même posé à cet effet un postulat anthropologique qui, espérons-le, ne fait pas l'unanimité de nos jours.

L'esprit cartésien et le développement technique du temps, dont en fait la poudre à fusil semble vouée à devenir l'argument le plus frappant, serviront comme base de comparaison pour prôner cette supériorité de l'un sur l'autre. Si l'Amérindien est une victime de premier plan de cette forme de pensée et des conséquences qui en 
découleront, il ne faudrait surtout pas oublier que ce sera aussi le lot de plusieurs autres peuples (autochtones en particulier) et d'une moitié de l'humanité représentée par la femme. Cette dernière n'est-elle pas intuitive et d'un raisonnement douteux et n'a-t-elle pas eu droit à son âme que tout récemment, selon cette forme de pensée que j'oserais croire primitive?

L'évolution humaine est tributaire de ses croyances, et c'est sur la base de celles-ci que s'élabore un scénario aux répercussions plus ou moins heureuses. II ne faut cependant pas se leurrer, il faudra un certain temps avant que le colonisateur " n'impose " ses lois et vérités et ne parvienne au résultat escompté : déposséder l'Amérindien. II faudra prendre en considération l'évolution du rapport de force sur le plan économique et militaire (ou guerrier) entre les groupes en présence.

\section{Des alliances à l'appropriation du territoire}

Pour bien saisir l'évolution des forces en présence, la lecture d'une analyse de P. Dionne, parue dans la Revue du Barreau, est à recommander (Dionne, 1991 : 130-144). Cette analyse montre comment, dans un premier temps, les Amérindiens, de par leur occupation et leur position stratégiques sur un territoire qu'ils connaissent bien, doivent forcément être abordés par Français et Anglais à titre d'alliés commerciaux et militaires, et non comme entités à s'aliéner. Les Amérindiens sont courtisés et chacun veut les gagner à sa cause. C'est l'époque des alliances où tous cherchent leur intérêt.

Mais avec l'établissement de la domination anglaise sur les Français, la rivalité commerciale dont tiraient avantage les Amérindiens commence à s'estomper. Le jeu des alliances devient désuet et se fait jour la possibilité de traiter différemment avec les Amérindiens. Cette situation donne lieu à de l'exploitation sur le plan commercial, ainsi qu'à une appropriation du territoire sans égard aux autochtones.

L'occupation du territoire se poursuivra malgré les doléances des Amérindiens, et il en résultera une montée d'insatisfaction qui prépare le soulèvement sous la conduite du chef Pontiac. À Londres, à ce moment, le "Board of Trade " a entendu parler d'un soulèvement imminent. Cela est perçu comme une menace à l'exercice du commerce et comme un conflit guerrier potentiel pouvant s'avérer très coûteux, menant même à une perte possible. Il s'empresse de recommander au roi de prendre des mesures pour arrêter cette montée d'insatisfaction et rassurer les Amérindiens. C'est ce qui servira de contexte à la Proclamation royale de 1763 qui donne des garanties aux Amérindiens sur leur territoire et sur l'exercice de certaines activités. 
Avant d'en citer un court extrait, rappelons seulement que la légalité de cette proclamation fut confirmée à diverses reprises et plus particulièrement par la Cour suprême du Canada qui lui reconnaît la force d'une loi du parlement. Cela permettra d'avoir une meilleure idée de ce sur quoi s'appuient les Amérindiens d'aujourd'hui, entre autres, lorsqu'ils parlent de leurs droits.

And whereas it is just and reasonable and essential to our interest, and the security of our Colonies, that the several nations or tribes of Indians with whom we are connected, and who live under our protection, should not be molested or disturbed in the possession of such parts of our dominions and territories as, not having been ceded to or purchased by Us, are reserved to them, or any of them, as their hunting grounds.

Pour la sécurité des colonies anglaises, comme le stipule cette proclamation, le gouvernement impérial se devait de donner assurance aux autochtones qu'il entendait mettre fin, une fois pour toutes, aux empiétements dont ils étaient victimes de la part des colons.

Bien que des directives aient été émises par le roi pour faire observer la Proclamation royale, l'accaparement du territoire reprendra finalement et plus le temps passera, moins les gouvernements en place ne respecteront ni l'esprit, ni la lettre de cette proclamation. Pratiquement, elle n'aura eu pour résultat que de calmer le soulèvement amérindien, le temps qu'il fallait pour accroître la présence militaire et diminuer la menace amérindienne à ce chapitre. Elle aura aussi pour effet, bien sûr, de créer un énorme écart entre le droit amérindien jeté sur papier et son exercice véritable, tel que rendu possible par la réalité.

\section{Le paradoxe d'une loi contre ses auteurs}

Ne peut-on s'empêcher de remarquer le bon paradoxe, lorsqu'un premier ministre rappellera qu'il n'y a qu'une seule loi pour tous, en sachant que précisément et de toute évidence l'appropriation d'une grande partie du territoire s'est faite en contravention avec cette loi ? Nous n'en sommes pas à un paradoxe près, ne serait-ce qu'en fonction de certains textes à caractère légal, dont certains groupes d'Amérindiens tirent aujourd'hui des déductions précises. Que ceux-ci y décèlent une autorisation à mener certaines actions n'expliquerait-il pas pourquoi les gouvernements n'interviennent pas ipso facto sur le plan de la loi, plutôt que de chercher à entraver et discréditer ces actions aux yeux de l'opinion publique?

L'homme a beau faire sa loi, il a beau vouloir l'ériger en absolu et établir des mécanismes pour faire la loi, il s'agit d'un exercice com- 
plexe qui peut entraîner des situations gênantes. Voilà qu'en vertu de cette loi, des actions et moyens qu'il voudrait se voir réserver seraient reconnus à d'autres. Quelle situation embarrassante et difficile à négocier! Comment donc réparer cette faille, sinon en espérant que l'autre commette des erreurs et des fautes, se discrédite devant l'opinion publique, créant ainsi l'occasion de colmater la brèche dans la loi. Le groupe concerné devra alors apprendre que la justice humaine sait s'adapter à la règle du deux poids, deux mesures et que son plus grave péché est peut-être d'avoir trop voulu copier son adversaire, en ce qui a trait à certaines pratiques et prérogatives. Machiavel, le voit-on, n'étant pas né d'hier, ses adeptes ont acquis une longue pratique de ses enseignements.

\section{La longue période d'infériorisation}

La réduction au minimum du rôle des Amérindiens et leur exclusion de l'exercice du pouvoir se poursuivront donc et on aura droit dans la suite historique aux deux "peuples fondateurs ». Les Amérindiens n'auraient pas de contribution qui leur vaille mention et écoperont plutôt d'une loi leur octroyant un statut de mineur. Ce sera le début du paternalisme mésadapté qui permettra de pousser plus à fond l'appropriation et la domination. Les Amérindiens seront bientôt confinés à des territoires de plus en plus restreints; ils seront parqués dans des réserves pour y être protégés en attendant le parachèvement de leur assimilation.

Une dépossession non légalisée et une longue période d'infériorisation systématique par ses hôtes de jadis seront le lot de l'Amérindien pour les décennies à venir. Malgré la situation, celui-ci résiste à son assimilation, mais cela ne peut se faire que par une difficulté d'adaptation au nouveau contexte de vie. II se retrouve dans un dilemme : s'identifier à une culture qui perd son espace vital ou participer à un monde qui exige de lui qu'il renonce à son identité distincte. II semblerait que ce soit le sort commun des peuples autochtones de se trouver placés devant un tel choix. Les cultures dominantes changent et leurs membres participent à la définition de cette transformation. Les individus présentent forcément des différences marquées $d^{\prime}$ avec leurs ancêtres, sans y reconnaître une perte d'identité ou une acculturation. II n'en est rien pour les autochtones, privés d'une pleine participation à la définition de leur avenir et traités d'acculturés, dès qu'ils se différencient un peu trop de leurs ancêtres. Ce cul-de-sac leur sera continuellement présenté, tant par le commun des mortels que par les scientifiques de tout acabit; et le pire, c'est que cette voie sans issue sera longtemps et abondamment adoptée par les Amérindiens 
eux-mêmes. Pas de continuum pour les cultures autochtones, seulement une image du passé... un moment fixé dans le temps.

Nous verrons disparaître progressivement cette allure fière et noble de nos ancêtres qui portaient la tête haute et le regard droit. Elle sera remplacée par un regard fuyant chez les générations qui suivront, comme si plusieurs Amérindiens n'osaient plus établir le contact avec leurs hôtes. L'âme humaine est sensible aux ambiances et se laisse impressionner par le milieu où elle évolue. Le jeune Amérindien sera forcé d'aller au pensionnat, à l'école non autochtone où il ne peut parler sa langue et apprend I'histoire " sans doute très véridique » du Canada. Bon gré, mal gré, l'âme amérindienne sera baignée dans une culture qui la coupe de ses pratiques spirituelles et dévalorise les siens. Une culture qui veut occuper tout l'espace et créer l'ambiance prédominante. Une âme a beau conserver une sorte de mémoire et nostalgie plus ou moins latente pour son passé, elle peut difficilement ne pas être altérée par une telle expérience.

Mais si l'âme amérindienne est, selon cette hypothèse, altérée, pour ne pas dire ébranlée, dans les circonstances, rappelons que le non-autochtone a aussi subi forcément une influence amérindienne. La contribution des Amérindiens à l'établissement de ce pays et leur influence sur les us et coutumes de ses habitants est évidente. Cela nous amène à l'obligation de reconsidérer le sens réel du terme assimilation et à lui donner un contexte.

Pour Piaget, l'assimilation constitue une des deux composantes essentielles du processus d'adaptation, l'autre composante étant l'accommodation (Piaget, 1967 : 9-24). Comment ce terme d'assimilation en est-il alors venu à créer, avec raison sans doute, autant de crainte chez les peuples ? C'est qu'en fait, lorsque deux entités en présence sont véritablement capables d'un respect profond pour leurs différences respectives, chacune est alors disposée à s'accommoder pour tenir compte de la réalité de l'autre. Il peut s'ensuivre une alternance du processus d'assimilation et d'accommodation pour produire une transformation respectueuse des deux entités en présence. Voilà peutêtre l'exercice qui n'a pas encore eu véritablement lieu et auquel I'humanité est toujours conviée, sous peine d'une inadaptation qui prend des allures inquiétantes. II y a déjà bon nombre d'années que l'Amérindien observe certaines actions fort destructrices de l'homme blanc et que cela lui fait dire que ce dernier n'aura besoin d'aucune aide pour courir à sa propre perte, s'il s'obstine sur cette voie.

Maintenant que nous avons tenté de définir le contexte et ses principales caractéristiques, voyons si nous pouvons dégager une liste de symptômes associés à l'oppression amérindienne. Il est fort probable que ces symptômes présentent de grandes affinités avec ceux qu'éprouvent d'autres groupes soumis à l'oppression. Si cela permet 
d'établir des sympathies et de sortir un peu de la tour de Babel, où le diviser pour régner prend tout son sens, alors un pas dans la bonne direction aura été accompli. Pensons que l'histoire récente n'a pas seulement réduit l'Amérindien à la dépendance économique, mais qu'elle en a fait autant pour de nombreux autres peuples ou nations, sans oublier les femmes et d'autres catégories de personnes. Nous laissons à chacun le soin de discriminer ce qui est particulier aux Amérindiens sur la toile de fond commune du phénomène de l'oppression.

\section{Symptômes d'oppression chez les Amérindiens}

1. Dépendance économique et pauvreté sont au premier rang des symptômes de cette oppression. Nous l'avons vu, la dépossession et la perte d'un espace vital à la culture ont fait leurs ravages. Puis, les possibilités d'adaptation rattachées trop longtemps à une menace à I'identité distincte ont créé une marginalisation extrême. II en résulte un fort taux de prestataires d'aide sociale et de compensations qui accentuent davantage la marginalisation plutôt que de vraiment combattre efficacement la pauvreté et l'absence de possibilités de développement socio-économique à l'échelle des communautés. Qui plus est, ces compensations sont parfois l'occasion de réactions agressives pour certains non-autochtones qui peuvent se sentir lésés ou pénalisés. Dépossession qui donne lieu à des enjeux dans un cadre législatif où la loi est tantôt implorée, mais toujours ambiguë et d'un arbitraire absolu.

2. Minimisation et humiliation sont parties intégrantes de l'oppression amérindienne. Sacrifice et dévalorisation d'une culture excessivement riche, au nom d'une pseudo-supériorité basée sur quelques critères du temps. Minimisation qui laisse ses marques et atteint le summum de sa perfidie, lorsqu'elle exerce son action sur de jeunes âmes particulièrement vulnérables à son influence.

3. Identité précaire et difficile à situer dans son rapport au contexte du moment, voilà une autre caractéristique de l'oppression des Amérindiens. Nous I'avons vu, les Amérindiens se voient refuser de participer, comme entité distincte, à l'exercice de pouvoir rattaché au devenir de ce pays. Puis, comme individus, ils ne peuvent se différencier de leurs ancêtres qu'au risque d'être déclarés acculturés. Jusqu'à récemment, ils ne pouvaient pas fréquenter une université sans renoncer à leur statut d'Amérindien. Une histoire, une identité, une culture arrêtées dans le temps. Un avenir sans devenir autre que sa disparition. Voilà le choix qui semble s'offrir aux Amérindiens. 
4. L'oppression consiste aussi à nous voir accuser d'être les premiers et seuls responsables de nos difficultés, en ne voulant pas nous adapter au changement. Accusation qui perd de son sens lorsque I'on comprend mieux les choix qui s'offrent.

5. L'oppression se trouve aussi dans la frustration de ne pas trouver cette troisième oreille capable de discerner le message latent derrière les manifestations de premier plan. C'est ce dialogue de sourds et même cette rivalité et agressivité entre groupes d'opprimés insensibles à leurs causes respectives et faisant cavalier seul. C'est subir la couverture de médias centrés sur le fait divers et manquant de temps ou d'intérêt, si ce n'est de capacité, pour discerner le contexte.

6. L'oppression, c'est de devoir démontrer l'évidence et d'avoir à se battre pour obtenir un strict minimum de respect et de droits, en ce qui regarde une participation active et décisionnelle à son avenir et dans ses propres institutions. Puis c'est, après avoir été exclu et minimisé, de devoir exceller avant d'avoir pu mettre en pratique et développer les aptitudes nécessaires à la tâche entreprise. De faire ses preuves dans des conditions précaires qui prennent peu en considération le processus approprié pour s'affranchir d'une longue période d'oppression; ce qui crée le risque d'un échec et la possibilité de confirmer "l'infériorisation théorisée " plutôt que de réparer les torts causés.

7. L'oppression, c'est aussi une action qui débouche sur une profonde rancune et agressivité devenant la toile de fond de l'existence de bon nombre d'opprimés. En contrepartie, chez les Amérindiens, il y a alors la possibilité d'une nostalgie et d'une sur-idéalisation d'une culture grandement sacrifiée, arrêtée dans un moment de son évolution et prenant difficilement racine dans un contexte qui pour sa part a grandement changé. Cela ne fait qu'accentuer un grand déchirement et la résurgence de sentiments négatifs (rancune, colère) possibles dans l'âme amérindienne qui alors paradoxalement se trouve privée de son bien le plus précieux : une ambiance empreinte de respect et de révérence.

8. Une dernière mais non moindre particularité de l'oppression amérindienne est sans contredit que nous sommes en présence d'un groupe opprimé qui possède des droits considérables sur papier. Droits que ce groupe ne peut faire respecter à cause d'une réalité qui s'est développée sans égard à ses droits, alors qu'il ne disposait pas de la force trinitaire mentionnée pour y remédier. La vérité serait-elle davantage véhiculée par la poudre à canon que par les textes de loi ? Néanmoins, il s'agit d'une condition propice à nourrir des attentes continuellement déçues, un dialogue qui cache son incongruité derrière le désir que le temps finisse par arranger les choses et un malentendu avec son cortège de frustrations et de confrontations. II s'agit aussi d'un processus qui a peut-être trop longtemps empêché de 
chercher ailleurs qu'à l'intérieur de ses paramètres et empêché d'envisager des solutions de rechange possibles, dans un climat de respect favorable à une telle démarche.

\section{Que font les Amérindiens?}

Si l'on se reporte au contexte d'aujourd'hui, la question se pose de savoir ce que font les Amérindiens pour améliorer leur condition. La partie la plus évidente de leur action est la revendication d'autonomie et de prise en charge. Ils revendiquent donc cette pleine participation aux institutions et processus de décision qui regardent leur avenir.

Cette demande de prise en charge, on la retrouve notamment dans le domaine des services sociaux. Assumer le contrôle des services sociaux s'adressant à leur population, cela semble bien évident. Eh bien ! détrompez-vous, car il y a les lois québécoises qui font obstruction. Il y a aussi une forte tendance à vouloir uniformiser et une crainte quasi viscérale des différenciations potentielles dont s'accommodent difficilement les structures en place. Comme conséquence, les Amérindiens ne se voient même pas reconnaître l'autorité légale pour accréditer des ressources d'hébergement à l'intérieur de leurs propres communautés, et on répond à leur demande d'autonomie en les laissant progressivement gérer des règles qu'ils n'ont pas l'autorité d'établir; dans la plupart des cas, ils n'ont même pas l'autorité de participer à leur élaboration.

Bien entendu, il faut reconnaître des divergences d'allégeances parmi les non-autochtones et l'existence de plusieurs sympathisants aux requêtes des autochtones. Ces sympathisants se retrouvent dans la population en général comme dans l'appareil gouvernemental. À tel point qu'il serait fort erroné de ne pas tenir compte de la montée de cette ouverture à l'autre et du positionnement différent qui en résulte dans le rapport entre autochtones et non-autochtones. Cependant, il ne faut pas croire que l'ancienne pensée soit morte et enterrée et il importe de se rappeler qu'il demeure encore bon nombre de fonctionnaires et de politiciens qui persistent à fonder tous leurs espoirs sur l'assimilation comme solution finale, perpétuant ainsi les fausses prétentions et les promesses rompues d'un processus devenu légendaire.

$\mathrm{Ce}$ droit de participation dont il est question, nous l'avons vu également s'exprimer dans le contexte de la Commission BélangerCampeau et dans celui des négociations du Lac Meech. Du premier, on retiendra l'argument qu'on ne veut pas faire d'exception. Argument pour le moins étonnant dans le cadre d'une commission qui se penche sur la nécessité de faire un cas d'exception à l'intérieur du Canada. Du second cas, on retiendra l'incapacité de mettre en évidence cette 
requête bien simple d'une demande de participation et d'intégration au processus. Au nom du Canada, on y décèle surtout une entrave aux procédures et on s'offusque du manque de sympathie de ceux qu'on a, en l'occurrence, si bien méprisés.

Un autre aspect de l'action des Amérindiens pour améliorer leur sort consiste en la revalorisation de leur culture. Ayant pris conscience du processus débilitant auquel ils ont été exposés, certains Amérindiens espèrent pouvoir participer à une revalorisation de leur histoire comme peuple et comme humains. Ils rejoignent ainsi d'autres groupes à une époque qui, malgré ses faiblesses à réparer les torts causés, permet tout au moins une plus grande expression et se montre plus perméable à une prise de conscience accrue.

Du côté un peu moins positif, on retrouve des manifestations de groupes qui affrontent violemment la société dominante et dont le conflit porte en bonne partie sur des prérogatives qu'ils se croient permises par certains textes de lois. Prérogatives que le gouvernement de la société dominante veut habituellement se réserver. On peut à la limite comprendre que la frustration et l'impuissance de l'opprimé débouchent sur de telles expressions, mais on ne peut aussi que le déplorer. Le déplorer, car cela montre bien à quel point l'âme amérindienne est entraînée loin de sa préoccupation première, sa vie spirituelle. Aurait-il pu en être autrement en certains cas, si l'on tient pour acquis que de dominer et d'inférioriser l'autre peut difficilement s'exercer sans créer de grandes frustrations? Les adeptes du pouvoir ne semblent pas y être encore parvenus et ils n'ont d'autre choix que $\mathrm{d}^{\prime}$ ajuster la nature de leur mensonge à celle des réactions de frustration qu'ils engendrent.

\section{Conclusion}

Pouvons-nous espérer un contexte d'évolution moins centré sur l'amour du pouvoir et de la domination, sur la cupidité et la compétition? Pouvons-nous croire en la possibilité d'un contrat social fondé sur d'autres prémisses, ou le scénario est-il déjà trop engagé et inflexible?

L'endettement ne serait-il que la forme la plus récente de l'expression de cette cupidité et compétition sans limite ? Devons-nous remettre l'argent aux cupides qui cultivent l'art de nous endetter à même cet argent et pousser un peu plus loin l'absurdité ? Sommes-nous invités à poser un peu moins la question du pourquoi pour la remplacer par celle du pour qui ? Cela permettrait-il de mieux saisir le vrai visage de ceux qui se cachent derrière le rideau de la mise en scène de l'oppression et observent les peuples et individus se quereller entre eux ? 
Songeons seulement aux grandes contradictions de la présente civilisation. En période de crise, la capacité de production n'est pas altérée, il faut seulement la freiner et créer ainsi une condition de relative pénurie. Puis, les économistes nous expliquent, bien convaincus dans un premier souffle, que l'économie se porte mal, car nous vivons au-dessus de nos moyens. Reprenant leur souffle, ils nous rapportent maintenant que l'économie se porte encore plus mal, car I'indice de consommation a chuté ce dernier mois. C'est à se demander si l'économie n'est pas en réalité une philosophie de l'absurde par excellence.

C'est ainsi que bien qu'une forme de surabondance ait été instaurée, cela n'empêche nullement de craindre les crises économiques et, entre celles-ci, de confiner un nombre effarant d'humains à la pénurie.

Nous sommes bien loin des états d'âme amérindiens de jadis. Bien loin de ce lien sacré à l'environnement, remplacé par une considération nouvelle qui prend ses fondements dans la peur de la pollution. Nous demeurons privilégiés de vivre dans un monde encore merveilleusement beau, malgré tout ce que l'homme lui fait souffrir de tensions.

Mais est-il vraiment question de distance? Si seulement nous nous permettions d'entrevoir, ne serait-ce qu'un instant, un monde s'affranchissant de la tour de Babel et des nombreuses rivalités qu'a engendrées cette humanité. Un monde au rendez-vous de cet effort d'accommodation auquel l'humanité est conviée et par lequel une attitude de respect véritable se cristalliserait telle une pierre précieuse.

Lorsque nos ancêtres entrevoyaient cet espoir de grande fraternité humaine, il en naissait un instant de magie. Alors, dans un cérémonial dont le sens profond est celui d'une véritable communion, ils fumaient le calumet, instrument consacré à ce rituel. Communiant au Grand Esprit pour bénéficier de son assistance, ils ne manquaient jamais de rappeler à chaque participant que nous sommes tous parents.

\section{Références bibliographiques}

DiOnNe, P. (1991). "Les postulats de la Commission Dorion et le titre aborigène au Québec : ving ans après, Revue du Barreau, tome 51, nº 1 : 127-171.

ÉLAN NOIR (1987). Les rites secrets des Indiens Sioux, textes recueillis par John Eps Brown. Paris : Le Mail.

PIAGET, J. (1967). La psychologie de l'intelligence. Paris : Armand Colin. 